\title{
Cryogenic Dielectrics and HTS Power Apparatus: Research at the University of Southampton
}

\author{
David Swaffield, Paul Lewin, George Chen and Jan Sykulski \\ Electrical Power Engineering Research Group, School of Electronics and Computer Science, University of \\ Southampton, Southampton, SO17 1BJ, UK
}

\section{Introduction}

Historically research into applications of low temperature superconductivity (LTS) (using multi-filament wires of NbTi for example) has produced coils of both complex size and shape which have found applications in such areas as nuclear magnetic resonance spectroscopy, magnetic resonance imaging and proton cyclotrons. However, LTS has not been successfully applied to electrical power devices mainly due to problems with reliability, high costs and complexities of cooling technology. On the other hand, high temperature superconducting (HTS) materials offer better thermal stability, reduced cooling costs (cf LTS) and improved reliability. HTS ceramic materials were discovered nearly twenty years ago and have conductivities, when superconducting, more than a million times greater than copper at room temperature. They operate reliably at liquid nitrogen $\left(\mathrm{LN}_{2}\right)$ temperatures and offer practical current densities twenty times greater than traditional copper windings. HTS materials present great potential for electrical power applications including cables, transformers, generators, fault current limiters and flywheels. It is well established that a major potential benefit of HTS power apparatus is that, compared to conventional designs, there are savings in both size and weight for the same rated power. In addition, there are lower losses and less environmental impact from nitrogen filled equipment compared with conventional mineral oil filled equipment. The development of HTS technology has presented several research challenges in terms of design, modelling and simulation as well as fundamental research into the behaviour of both solid and liquid dielectric materials at $\mathrm{LN}_{2}$ temperatures.

\section{Southampton HTS projects}

The University of Southampton has a long history of research into cryogenics and superconductivity. A central focus for work in recent years has been the design and construction of demonstration power devices as well as research into the behaviour of liquid nitrogen in the presence of electrical and thermal fields. For the acceptance of HTS power apparatus designs, they will have to be both economically competitive with conventional alternatives and reliable in operation. To achieve this, HTS designs must be optimised for performance, to minimise cost of plant and cost of operation and to ensure reliable performance. It is towards this goal that research has focussed at Southampton, both in the areas of electromagnetic design and dielectric research. HTS applications present serious electromagnetic modelling challenges because of the highly non-linear and anisotropic HTS tape material characteristics and because they are being applied in unconventional designs. This paper considers some of the challenges of designing HTS power apparatus and then discusses work to characterise solid dielectrics and $\mathrm{LN}_{2}$ at temperatures in the range 64-77 K.

\section{0 kVA demonstrator transformer design}

A successful small $10 \mathrm{kVA}$ demonstrator transformer design, manufacture and testing project was completed at the University of Southampton in 1999, Figure 1 [1]. A single phase design with copper primary and BSCCO-2223 superconducting tape pancake coil secondary was used to study the performance of tapes and allow the comparison with electromagnetic models developed. The large separation between the two coils, 
required to accommodate the cryostat, increases the radial flux densities and leakage reactance and so a three limb core design was used with both windings on the centre limb. The current rating of the secondary coil at a load of $10 \mathrm{kVA}$ is $40 \mathrm{~A}$.

The flux component normal to the broad face of the HTS tape must be minimised as this is critical to the current carrying capacity of the winding; for example to carry the peak current of 9.5 A per tape, flux density must be less than $15 \mathrm{mT}$, compared with $110 \mathrm{mT}$ for the parallel component. To achieve this, flux diverters manufactured of powdered iron epoxy composite were placed next to each end coil. Figure 2 shows field plots of the coils with and without the flux diverters in place.

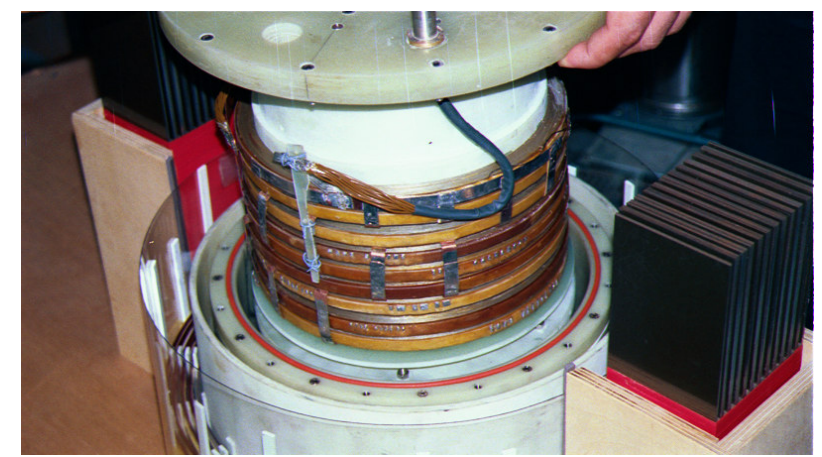

(a)

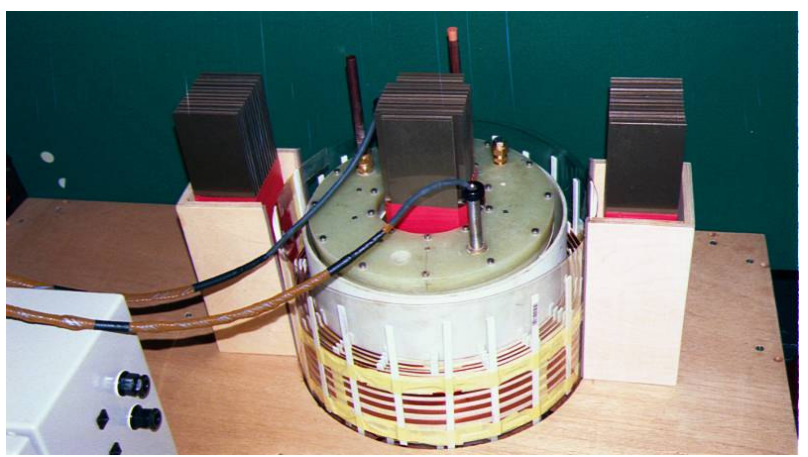

(b)

Figure 1, $10 \mathrm{kVA}$ demonstrator transformer a) HTS pancake coils shown with cryostat open, b) copper coil around closed cryostat, top yoke removed

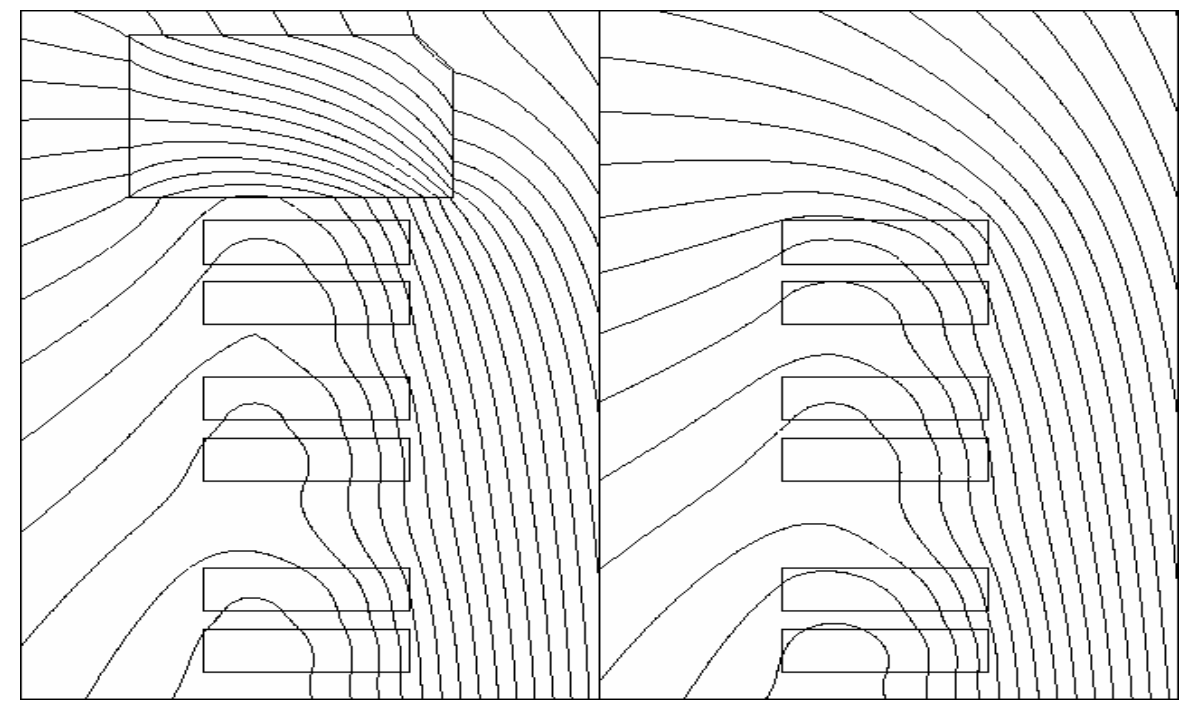

Figure 2, Magnetic field plots around HTS coils with and without flux diverters

Figure 3 shows the modelled and measured losses in the cryogenic region plotted against secondary current with and without the flux diverters. Tests were performed beyond the nominal design rating of the secondary. These plots validate the modelling approach developed to estimate the AC losses described in [2]. The losses were reduced to a half of the value with no flux diverters, showing also the benefits of good modelling as a design aid. 


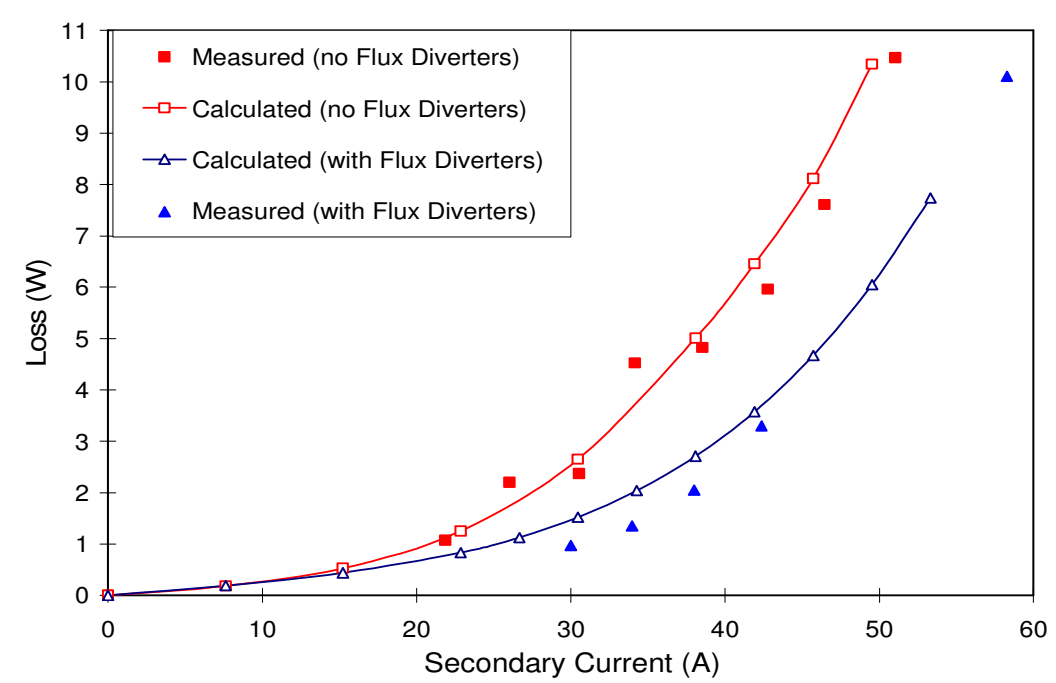

Figure 3, Measured and calculated losses with and without flux diverters

\section{MVA grid auto-transformer design study}

A design feasibility study was conducted to consider the technical and economic viability of a 240 MVA grid autotransformer. [3,4] The key parameters of the design studied are listed below;

kVA:

240,000

Normal volts:

$400 / 132 \mathrm{kV}$

Tappings:

$132 \mathrm{kV} \pm 15 \%$ in 14 steps

Line current at normal volts:

Diagram No:

$346 / 1054$ A

Guaranteed reactance:

Yy0 Auto

Rated current densities:

- Series winding* $=39.1 \mathrm{~A} / \mathrm{mm} 2$

- Common winding* $=36.9 \mathrm{~A} / \mathrm{mm} 2$

- Tap winding $=3.0 \mathrm{~A} / \mathrm{mm} 2$ (conventional)

*average over composite conductor section, comprising both superconducting and matrix materials

The principal feature of the design is the removal of the copper windings and their replacement by HTS coils. The HTS coils are less than $10 \%$ the size of copper equivalents. Thus an advantage is seen in the reduced size and weight of the transformer design, Table 1 . However, the inevitable result is windings of reduced mechanical strength, thus additional bracing is necessary to withstand the radial bursting force and axial compressive forces that occur during fault conditions. For the studied design the tap winding was chosen to be located outside the cryostat to reduce the thermal in-leak which would result from the multiple connections at ambient temperature. It is desirable to remove oil from the design to reduce the risk of fire and prevent environmental impact from spillage. To remove oil from the design completely the tap windings can be cooled by forced gas cooling.

The HTS tapes have a low thermal mass and are stable only over a small temperature range. The design is therefore vulnerable to through faults, requiring disconnection and cooling for several minutes after experiencing a fault. The transformer will survive the most severe fault if disconnected within $166 \mathrm{~ms}$, see Table 2. This is the major weakness of the HTS transformer design; however there is good overload capability. The main savings are on 'copper' losses, which lead to the HTS design releasing only $23 \%$ of the total losses when compared with a conventional transformer. 
Table 1. 240 MVA grid autotransformer size and weight comparison.

\begin{tabular}{|c|c||c|}
\hline Parameter & HTS & Conventional \\
\hline Core length $*$ & 88.5 & 100 \\
height $*$ & 82.4 & 100 \\
thickness $*$ & 100 & 100 \\
Window, height $*$ width $*$ & $70 \times 78.5$ & $100 \times 100$ \\
\hline Core weight $*$ & 80 & 100 \\
Winding weight $*$ & 6.3 & 100 \\
Tap winding weight $*$ & 100 & 100 \\
\hline Cooling of core & Forced N2 gas & ONAN/OFAF \\
and tap winding & Liquid N2 & ONAN/OFAF \\
Cooling of common & (with refrigeration) & \\
and series winding & & \\
& & \\
\hline
\end{tabular}

*shown as a percentage of conventional design equivalent.

Table 2. Technical features comparison.

\begin{tabular}{|c||c|c|}
\hline Parameter & HTS & Conventional \\
\hline Guaranteed \% reactance & 20 & 20 \\
B in core, T & 1.67 & 1.67 \\
J rated, rms, A/mm2 & 38 & 2.83 \\
\hline Rated loss, total * & 23 & 100 \\
\hline $\begin{array}{c}\text { Overload capability } \\
\text { Through fault capability, } \\
\text { pu (+ doubling transient), recovery } \\
\text { time without disconnection } \\
\text { Survival time at } 5 \text { pu } \\
\text { (+ doubling transient) }\end{array}$ & 2 pu, many hours & $1.3 \mathrm{pu}, 6 \mathrm{hrs}$ \\
& $1.5 \mathrm{pu}, 30 \mathrm{~min}$ \\
\end{tabular}

Capital and operational expenditures were assessed and first-cost savings calculated, based on a discount period of 10 years at $9.5 \%$ per year [4]. Savings of $36 \%$ as compared to a conventional copper winding transformer were predicted. However, this was performed under the assumption that the transformer was $100 \%$ loaded all of the time. This assumption is unrealistic, indeed on the national grid in England and Wales the load factor of transformers is typically only $23 \%$. The application of HTS transformers therefore requires careful consideration. One proposal put forward was to use a HTS unit to replace one of two conventional transformers, of the size of that studied, running in parallel circuits on dual (main and reserve) circuits. This would enable a higher load factor for the HTS transformer, thereby achieving the potential cost savings. Alternative applications might be considered where transformers have a high load factor, for example substations at the point of generation.

\section{Cored generator design}

Many conceptual HTS designs were proposed, and small demonstrator generators built with BSCCO tapes, but most operate between 25 and $30 \mathrm{~K}$. The advantage of lower temperature cooling is that the critical 
currents and magnetic fields are an order of magnitude greater than at $78 \mathrm{~K}$ (i.e. $\mathrm{LN}_{2}$ temperatures). Since the magnetic field can be increased it is possible to have a core-less design. The disadvantage, as compared to a $\mathrm{LN}_{2}$ design, is that Liquid Neon ( $\mathrm{LNe}$ ) or Helium gas $(\mathrm{GHe})$ has to be used as a cooling medium. This leads to a greater complexity and higher cost of the refrigeration plant. Greater complexity results in worse reliability, comparable to LTS applications, and higher maintenance costs. Moreover, the thermodynamic efficiency is about eight times worse when working at 25-30K compared with $78 \mathrm{~K}$.

At Southampton these considerations have lead to the view that $\mathrm{LN}_{2}$ temperature designs should be pursued. The demonstrator generator designed is a $100 \mathrm{kVA} 2$ pole machine built to be operated under several cooling methods, including $81 \mathrm{~K}, 78 \mathrm{~K}, 65 \mathrm{~K}$ and $57 \mathrm{~K}$ using $\mathrm{LN}_{2}$ or liquid synthetic air and sub-cooled $\mathrm{LN}_{2}$ or liquid synthetic air [5]. A schematic diagram of the generator is shown in Figure 4. Liquid synthetic air (79\% nitrogen, $21 \%$ oxygen) has a melting point of $57 \mathrm{~K}$ and boiling point of $78 \mathrm{~K}$, thus offering a wider liquid range than with $\mathrm{LN}_{2}$. The use of a magnetic core rotor reduces the number of ampere-turns required by a factor of ten and significantly reduces the fields within the coils. In the generator built the rotor has been made of cryogenic steel (9\% Nickel). The rotor has 10 identical pancake coils made of BSCCO (Ag clad Bi2223), the length of the wires is approximately 10 lengths of $40 \mathrm{~m}$. Coils have been manufactured by a process of winding the fragile HTS tapes onto a former interleaved with insulating tape and then resin impregnated under vacuum. Each coil is then tested prior to rotor installation; this is to check that no damage has occurred during manufacture, for example by in-plane bending of tapes. The modular design allows any faulty coil to be replaced with ease.

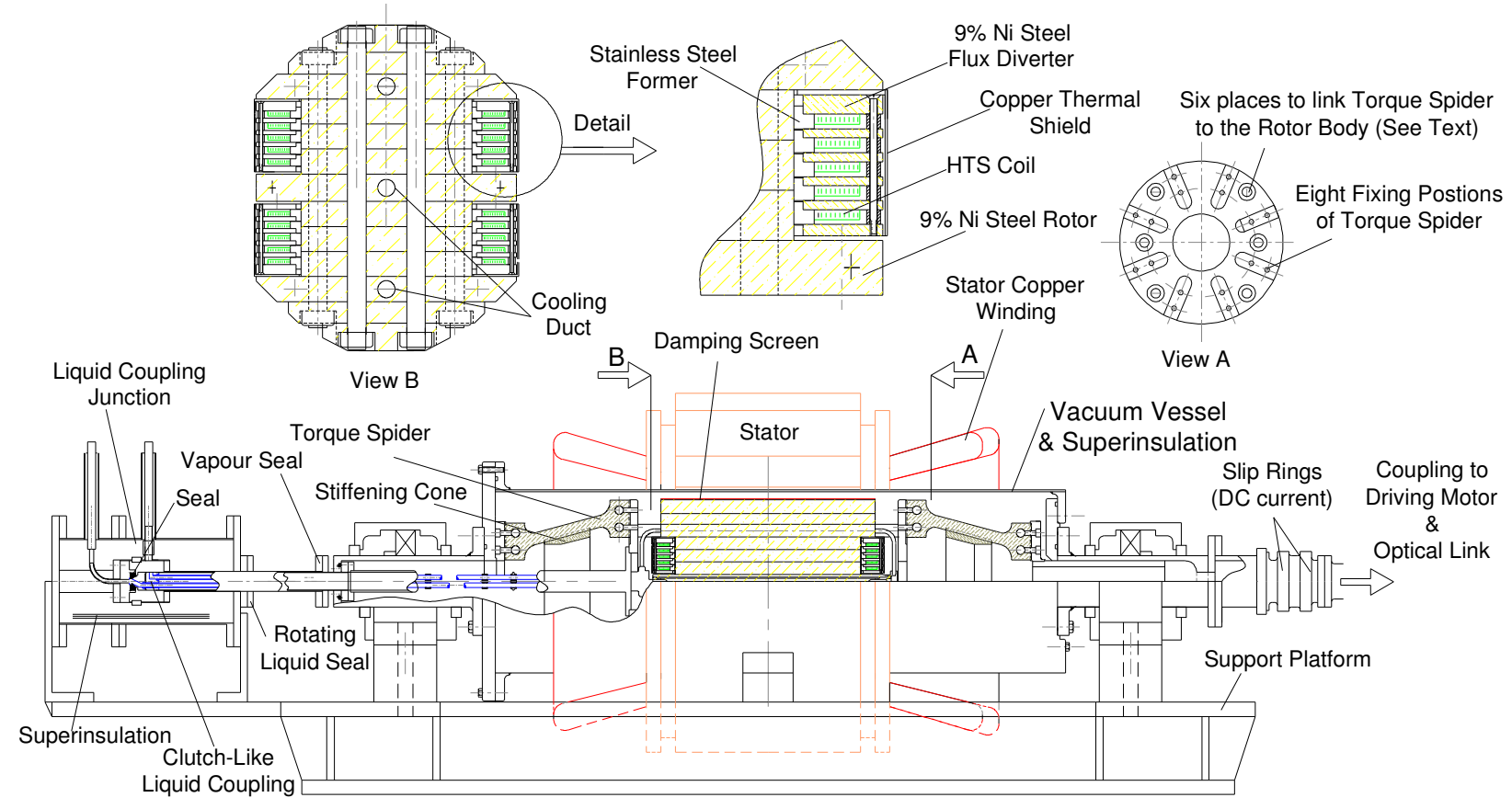

Figure 4. Southampton HTS $100 \mathrm{kVA}$ demonstrator generator design.

The pancake coils are placed onto two pole necks. The rotor shape is a hybrid design which uses flux diverters made of $9 \%$ Nickel steel placed between the coils to reduce the normal component of the magnetic field in the coil tapes by diverting flux around them and shaping the magnetic field profile in the gap, Figure 5. In this manner the normal component of the field in the coils has been reduced to $0.038 \mathrm{~T}$, while maintaining $0.66 \mathrm{~T}$ in the air-gap. Cooling is provided by a purpose built closed circuit liquid cryogen cooling system with pipe-network feeding liquid cryogen to the rotor body of the generator. To reduce thermal heat leak into the cryogenic region the mechanical linkages, between rotor core and the steel shafts, are made of fibreglass. A copper radiation screen is placed around the rotor core assembly to intercept heat radiating from the vacuum region and to limit the time varying fields in the superconducting winding. 
Electromagnetic modelling of the harmonics of the machine found that the significant contributions to losses are dominated by the $5^{\text {th }}$ harmonic and then to a smaller extent by $7^{\text {th }}$ harmonics, losses from $11^{\text {th }}$ and higher order harmonics are negligible. Overall load loss was calculated to be $2.319 \mathrm{~W}$. With predicted no load loss of $0.264 \mathrm{~W}$ the total power loss in the cryogenic region was $2.583 \mathrm{~W}$. Every $1 \mathrm{~W}$ lost at $77 \mathrm{~K}\left(\mathrm{LN}_{2}\right)$ represents a load of 15-25 W of installed refrigeration power. It is of note that equivalent figures at $27 \mathrm{~K}$ $(\mathrm{LNe})$ and $4 \mathrm{~K}(\mathrm{LHe})$ would be $200 \mathrm{~W}$ and $1000 \mathrm{~W}$ respectively. Thus the benefits of running at higher temperatures and minimising the loss at cryogenic temperatures are clear.

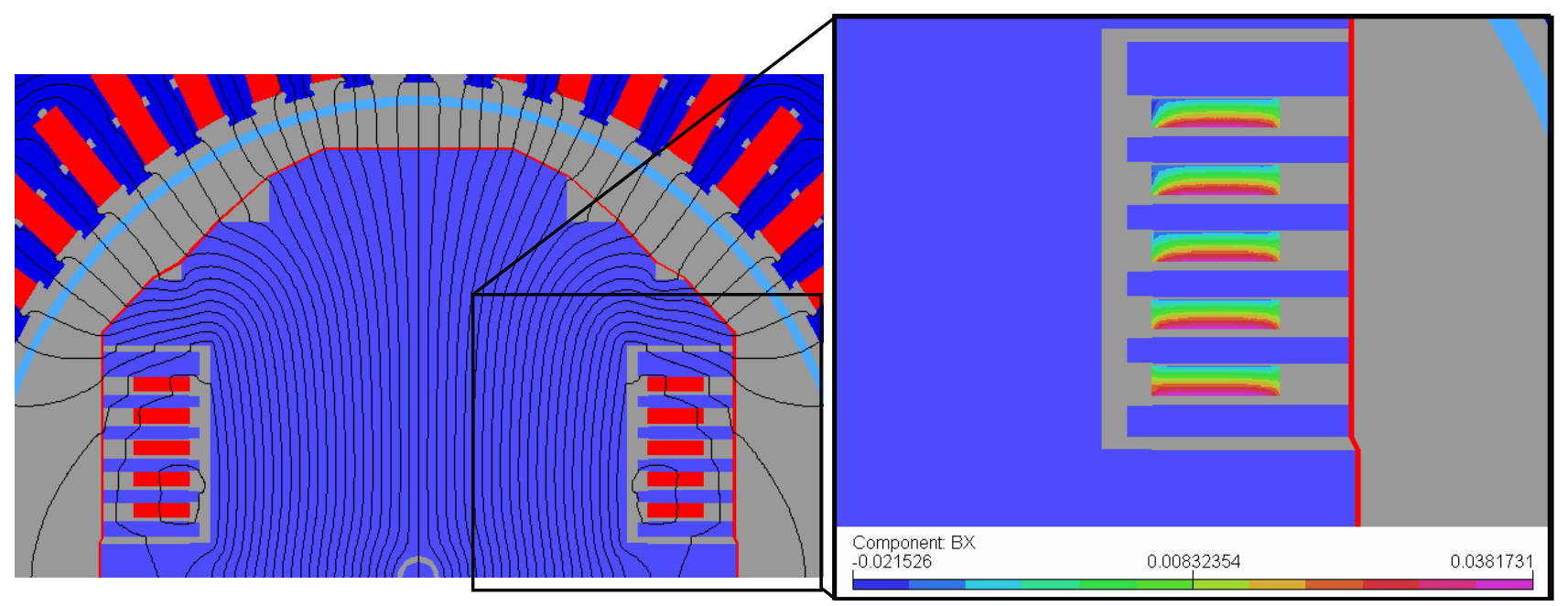

Figure 5. Flux lines in the superconducting rotor, showing one pole and (inset) the normal magnetic field in the coils

\section{Cryogenic dielectrics}

In order to realise the potential of HTS power applications they have to be economically competitive, technically viable and reliable in operation. The development of applications reported has demonstrated that the considerable design challenges of HTS equipment can be met and performance accurately modelled. Generally, power equipment design and manufacture projects have not always met with success and many of these have been due to dielectric failures. The motivation for the study of cryogenic dielectrics to ensure reliable operation of designs is therefore clear. Only by characterisation of dielectrics, solid, liquid and composite systems can reliability be designed into equipment. Knowledge of dielectric performance will be important where designs have to be optimised for savings by trading reduced cryogen volumes, reduced losses and higher temperatures against the dielectric performance. Towards this goal, work at Southampton has included the study of liquid nitrogen, pre-discharge characteristics, bubble nucleation and dynamics due to electrohydrodynamic (EHD) forces in electric fields.

\section{Bubble dynamics}

Bubbles are known to reduce the breakdown withstand level of $\mathrm{LN}_{2}$. Bubbles may be present due to trapped vapour on surfaces during filling, thermally or electrically induced. Nitrogen vapour bubbles in electric fields are subject to EHD forces governed by

$$
F v=q_{f} E-\frac{1}{2} E^{2} \nabla \varepsilon+\frac{1}{2} E^{2} \nabla\left(\rho \frac{\partial \varepsilon}{\partial \rho} E^{2}\right)
$$

Where $\mathrm{q}_{\mathrm{f}}$ is the free charge density, $\mathrm{E}$ is the electric field strength, $\varepsilon$ is permittivity and $\rho$ is density. With reference to (1), the first term is force due to Coulombic force of free charges and this is minimal for $\mathrm{LN}_{2}$ where the relaxation time is large and conduction poor [6]. 
In a study of bubbles nucleated from a heated cavity in a plane-plane electrode arrangement with an applied $\mathrm{dc}$ electric field; bubbles have been observed to remain on the surface for longer periods attaining a greater volume at detachment and larger aspect ratio; elongating in the direction of the applied electric field. Such an increase in vapour volume will be detrimental to the breakdown withstand level for two reasons, firstly field enhancement due to the bubble profile and different relative permittivity of the vapour and liquid phase, and second due to the reduced breakdown strength of the vapour as compared to $\mathrm{LN}_{2}$. The application of an ac field caused the bubble to oscillate in time with the applied field demonstrating the force is due to the dielectrophoretic and electrostrictive forces that are the second and third terms (1). In another study for bubbles rising under buoyancy force between rod-plane electrodes the bubble column is seen to move away from the region of high electric stress, at the rod electrode [7]. Thus reducing the threat to bubble aided breakdown, the design of the liquid dielectric and electric fields is therefore important from the point of view of designing out potential causes of failure.

\section{Partial discharge}

The application of high electric fields to LN2 can cause the initiation and propagation of density change streamers. To study the development of streamers a technique to synchronously record electric discharge signals with imaging of streamers using a high-speed digital camera has been developed [8]. Using a pointplane sample with composite LN2 and solid dielectric, streamers are initiated from a tungsten needle for three solid barrier materials, including a glass fibre reinforced resin (10G/40), a cotton fibre reinforced resin and polytetrafluroethyline (PTFE) $[6,8,9]$. A partial discharge is recorded for every streamer and discharges have been recorded for $50 \mathrm{~Hz}$ ac voltages, a range of hydrostatic pressures and liquid temperatures. The appearance of typical streamer shapes was confirmed for liquid nitrogen, both filamentary and bush-like streamers have been observed. Peculiar to $\mathrm{LN}_{2}$ both streamer shapes can be observed to occur for each polarity of needle tip. Although the negative tip initiated discharge occurs more frequently than the positive tip discharge for the same voltage; the positive tip will typically initiate a larger discharge event and a larger streamer growth is observed, often bridging the liquid part of the composite insulation system terminating on the solid dielectric surface. Erosion to the solid dielectric surface has been observed and will be further characterised in future studies. General trends with voltage, pressure and temperature have been established. The number of discharges will increase with applied voltage and the discharges advance in phase indicating the storage of charge within the system. Increasing pressure or reducing temperature has the opposite effect of reducing numbers of discharges charge magnitude and retarding the discharges on the phase. It could be argued that increasing the pressure be used as a strategy to reduce the likelihood of discharge and failure. However this may be undesirable as it requires greater mechanical strength of designs to safely hold the pressure and moves the design temperature up the saturation curve; the result is a higher working temperature and reduced HTS tape performance. By a similar argument reducing the operational temperature will reduce the risk of dielectric failure and improve the performance of the HTS tapes. This design decision must be taken with consideration of the effect on thermodynamic efficiency of refrigeration and operational cost.

\section{Summary and Conclusions}

This paper has presented the areas of research at the University of Southampton. HTS power apparatus must be economically competitive and technically viable which includes the need for reliability, in order to be accepted into service. To maximise the potential savings of HTS power equipment, the view taken at Southampton has been to design apparatus to operate at $\mathrm{LN}_{2}$ temperatures, thus greatly reducing the refrigeration load, cost and complexity. Several demonstrator projects have been undertaken including the design, manufacture and testing of a $10 \mathrm{kVA}$ transformer demonstrating the performance of HTS tapes and the satisfactory use of electromagnetic models to calculate losses, a key design parameter. In addition, a $100 \mathrm{kVA}$ cored generator has been successfully built and the design of a coreless design is the focus of the current research effort under a recently awarded EPSRC grant. With the recent progress toward high voltage HTS apparatus, for example cables and transformers, the need to develop a good understanding of cryogenic dielectrics is hastened. To this end fundamental studies have been undertaken to examine the bubble dynamics in electric fields and partial discharge and streamer behaviour within liquid nitrogen. Partial discharge behaviour has been characterised for $50 \mathrm{~Hz}$ ac for the working parameters of voltage, pressure and 
temperature. In future a focus of research effort on greater understanding of the performance, including erosion characteristics, of solids at cryogenic temperatures will be undertaken.

\section{References and Bibliography}

References along with an additional bibliography covering recent work at Southampton are included below. For full details of published work see http://eprints.ecs.soton.ac.uk

\section{HTS Transformers}

1. Sykulski, J. K., Goddard, K. and Stoll, R. L. (1999) High temperature superconducting demonstrator transformer: design considerations and first test results. IEEE Transactions on Magnetics 35(5):pp. 3559-61.

2. Sykulski, J. K., Goddard, K. F. and Stoll, R. L. (2000) A method of estimating the total AC loss in a high-temperature superconducting transformer winding. IEEE Transactions on Magnetics 36(4):pp. 1183-7.

3. Sykulski, J. K., Beduz, C., Stoll, R. L., Harris, M. R., Goddard, K. and Yang, Y. (1999) High temperature superconducting power transformers: conclusions from a design study. IEE Proceedings, Electrical Power Applications 146(1):pp. 41-52.

4. Sykulski, J. K. (2004) Superconducting Transformers. In Proceedings of Advanced Research Workshop, pages pp. 55-60, Vigo, Spain.

Sykulski, J. K., Rotaru, M. and Stoll, R. L. (2000) 2D modelling of field diffusion and AC losses in high temperature superconducting tapes. IEEE Transactions on Magnetics 36(4):pp. 1178-82.

Sykulski, J. K., Stoll, R. L., Beduz, C., Power, A. J., Goddard, K. F. and Al-Mosawi, M. K. (2000) The design, construction and operation of high temperature superconducting transformers - practical considerations. In Proceedings of 38th Session of the International Conference on Large High Voltage Electric Systems (CIGRE), Paris, 27th Aug-1st Sep 2000.

Goddard, K. F., Sykulski, J. K. and Stoll, R. L. (1999) A method of estimating the total AC loss in a high-temperature superconducting transformer winding. In Proceedings of COMPUMAG, pages pp. 66-7.

Goddard, K. F., Sykulski, J. K. and Stoll, R. L. (1999) A new approach to modelling dominant AC loss in HTS superconducting solenoidal windings. IEEE Transactions on Magnetics 35(3):pp. 1195-8.

\section{HTS Generator}

5. Mosawi, M. K., Beduz, C., Goddard, K. F., Sykulski, J. K., Yang, Y., Xu, B., Ship, K. S., Stoll, R. and Stephen, N. G. (2002) Design of a 100 kVA high temperature superconducting demonstration synchronous generator. Physica C 372-6(P3):pp. 1539-1542

Ship, K. S., Goddard, K. F. and Sykulski, J. K. (2005) Two dimensional finite-element simulation of a high temperature superconducting synchronous generator during three-phase short-circuit fault condition using full transient non-linear rotating machine model, in Wiak, S., Krawczyk, A. and Trlep, M., Eds. Computer Engineering in Applied Electromagnetism, chapter 21, pages pp. 355-358. Springer. 
Ship, K. S. and Sykulski, J. K. (2004) Field simulation studies for a high temperature superconducting synchronous generator with a coreless rotor. IEE Proceedings, Science, Measurement and Technology 151(6):pp. 414-418.

Al-Mosawi, M., Xu, B., Beduz, C., Goddard, K., Sykulski, J. K., Yang, Y., Stephen, N. G. and Stoll, R. (2003) Recent progress of 100kVA High Temperature Superconducting Generator. In Proceedings of 6th European Conference on Applied Superconductivity EUCAS 2003, Sorrento, Italy.

Ship, K. S., Goddard, K. F. and Sykulski, J. K. (2002) Field optimisation in a synchronous generator with high temperature superconducting field winding and magnetic core. IEE Proceedings, Science, Measurement and Technology, 149(5):pp. 194-198.

\section{Liquid Nitrogen}

6. Swaffield, D. J. (2005) Effect of liquid nitrogen bubble dynamics on insulation performance for high temperature superconducting power apparatus. PhD Thesis, University of Southampton, 2005

7. Wang, P., Swaffield, D. J., Lewin, P. L. and Chen,G. (2005) Thermal bubble dynamics in cryogenic liquids under non-uniform fields on superconducting power apparatus. In Proceedings IEEE Conference on Electrical Insulation and Dielectric Phenomena, Nashville Tennessee USA

8. Swaffield, D. J., Lewin, P. L., Chen, G. and Swingler, S. G. (2004) Variable Pressure and Temperature Liquid Nitrogen Cryostat for Optical Measurements with Applied Electric Fields. Journal of Measurement Science and Technology 15(11):pp. 2325-2332.

9. Swaffield, D. J., Lewin, P. L., Tian, Y., Chen, G. and Swingler, S. G. (2005) Characterisation of surface partial discharge behaviour in liquid nitrogen. In Proceedings of IEEE International Conference on Dielectric Liquids, pages pp. 143-146, Coimbra, Portugal.

Swaffield, D. J., Lewin, P. L., Tian, Y., Chen, G. and Swingler, S. G. (2005) Partial Discharge Characterisation in Liquid Nitrogen Composite Systems. In Proceedings of IEEE International Conference on Dielectric Liquids, pages pp. 139-142, Coimbra, Portugal.

Swaffield, D. J., Lewin, P. L., Tian, Y., Chen, G. and Swingler, S. G. (2004) Characterisation of partial discharge behaviour in liquid nitrogen. In Proceedings of IEEE International Symposium on Electrical Insulation, pages pp. 135-138, Indianapolis, Indiana, USA.

Swaffield, D. J., Lewin, P. L., Chen, G. and Swingler, S. G. (2003) The influence of bubble dynamics in liquid nitrogen with applied electric fields on superconducting apparatus. In Proceedings of 13th International Symposium on High Voltage Engineering, pages CD-ROM, Delft, Netherlands. 